Supporting Information for:

\title{
Triplet Energy Transfer from Perovskite Nanocrystals Mediated by Electron Transfer
}

Xiao Luo, ${ }^{1,2 \dagger}$ Guijie Liang, ${ }^{3+}$ Yaoyao Han, ${ }^{1,4}$ Yulu Li, ${ }^{1}$ Tao Ding, ${ }^{1}$ Shan He, ${ }^{1}$ Xue Liu ${ }^{1}$ and Kaifeng $W u^{1 *}$

${ }^{1}$ State Key Laboratory of Molecular Reaction Dynamics and Dynamics Research Center for Energy and Environmental Materials, Dalian Institute of Chemical Physics, Chinese Academy of Sciences, Dalian, Liaoning 116023, China

${ }^{2}$ School of Optoelectronic Science and Engineering, University of Electronic Science and Technology of China, Chengdu, Sichuan 610054, China

${ }^{3}$ Hubei Key Laboratory of Low Dimensional Optoelectronic Materials and Devices, Hubei University of Arts and Science, Xiangyang, Hubei 441053, China

${ }^{4}$ University of the Chinese Academy of Sciences, Beijing 100049, China

\section{Content list:}

Figures S1-S13

Experimental Methods

Kinetics fitting

Table S1-S2 

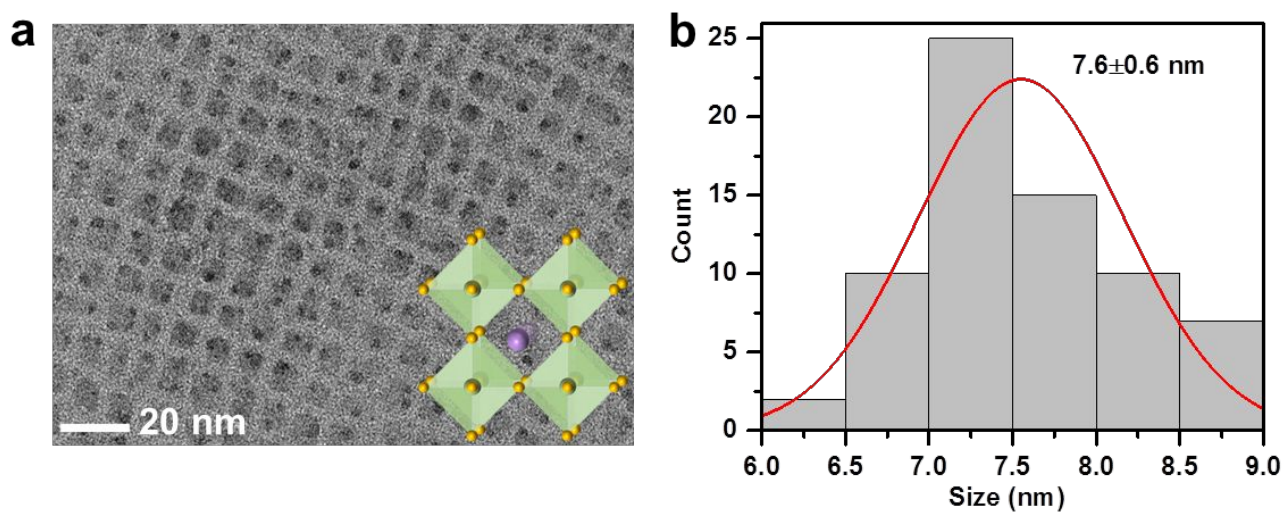

Figure S1. (a) TEM image of $\mathrm{CsPCl}_{3} \mathrm{NCs}$ used in the main text. (b) Statistic histogram of the edge lengths of the NCs in (a).
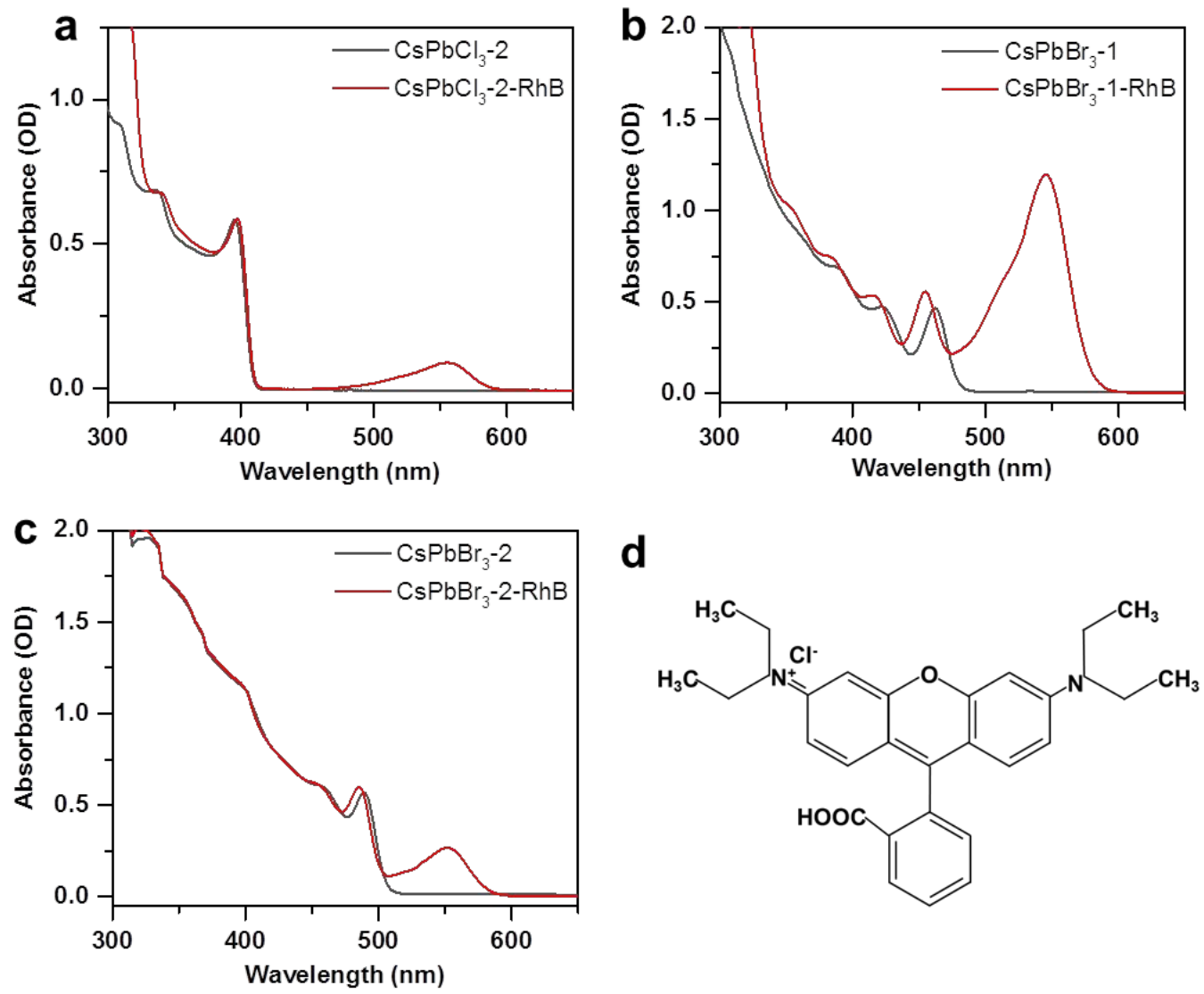

Figure S2. Absorption spectra of another $\mathrm{CsPbCl}_{3} \mathrm{NC}$ sample, (a) $\mathrm{CsPbCl}_{3}-2$, and two $\mathrm{CsPbBr}_{3} \mathrm{NC}$ samples, (b) $\mathrm{CsPbBr}_{3}-1$ and (c) $\mathrm{CsPbBr}_{3}-2$. According to our previously established sizing curve ${ }^{1}$, the edge lengths of $\mathrm{CsPbBr}_{3}-1$ and $\mathrm{CsPbBr}_{3}-2$ are $\sim 4$ and $9 \mathrm{~nm}$, 
respectively. Note that the absorption spectra of $\mathrm{CsPbBr}_{3} \mathrm{NCs}$ are slightly blue-shifted upon $\mathrm{RhB}$ adsorption, because of anion exchange between $\mathrm{Br}^{-}$in the lattice and $\mathrm{Cl}^{-}$in the $\mathrm{RhB}$ molecule. The molecular structure of $\mathrm{RhB}$ is shown in (d).

a

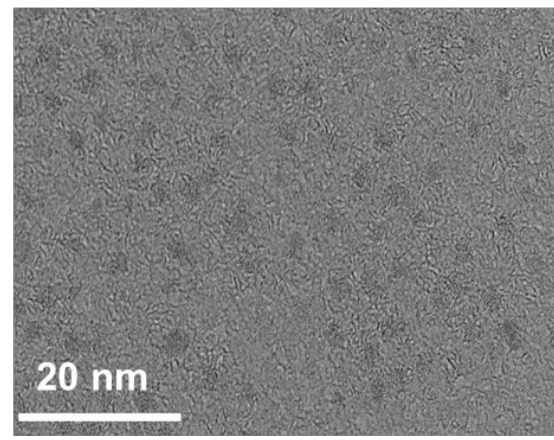

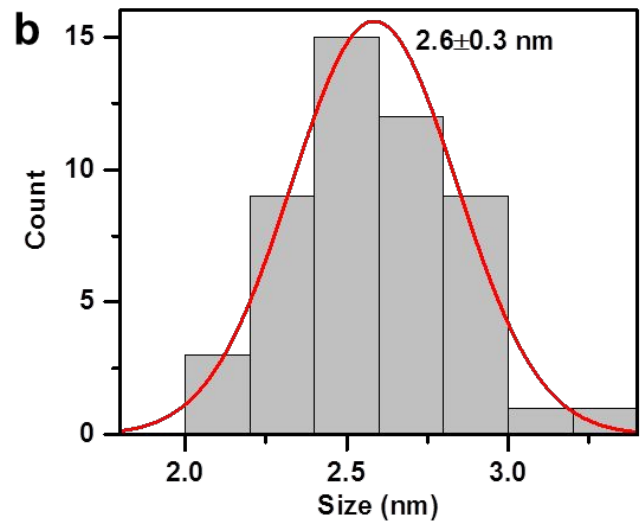

Figure S3. (a) TEM image of CdS NCs used in the main text. (b) Statistic histogram of the diameters of the NCs in (a).
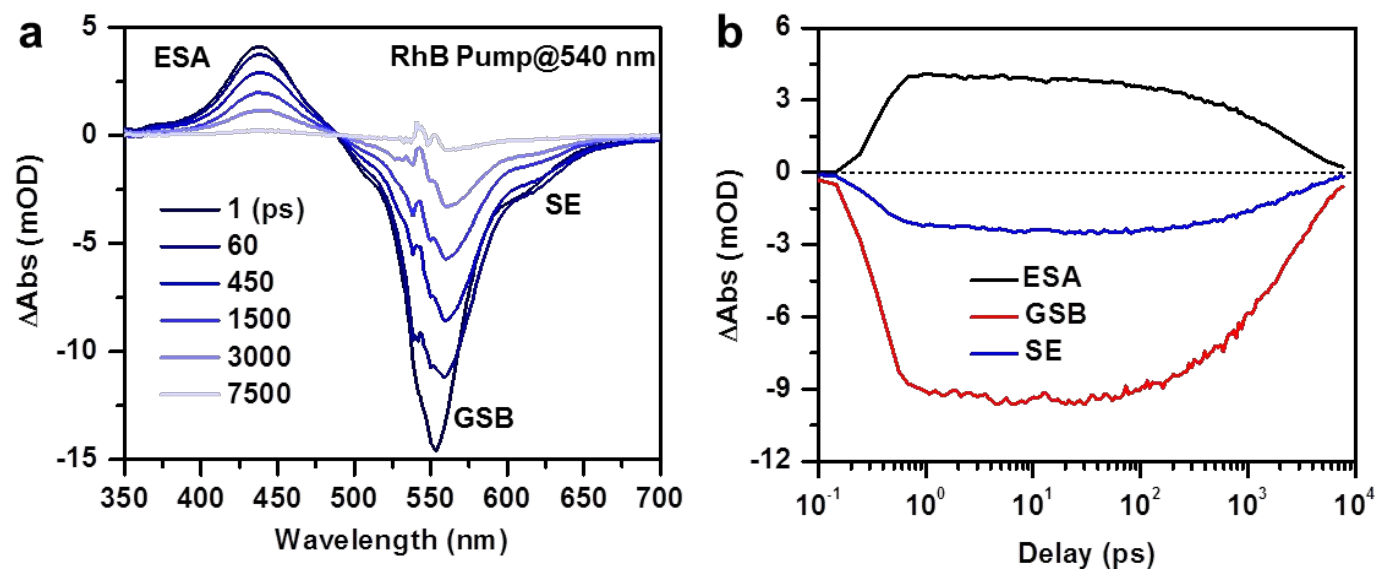

Figure S4. (a) TA spectra of $\mathrm{RhB}$ in ethanol excited at $540 \mathrm{~nm}$. The excited state absorption (ESA), ground state bleach (GSB) and stimulated emission (SE) features are indicated. (b) TA kinetics probed at the ESA, GSB and SE features. 

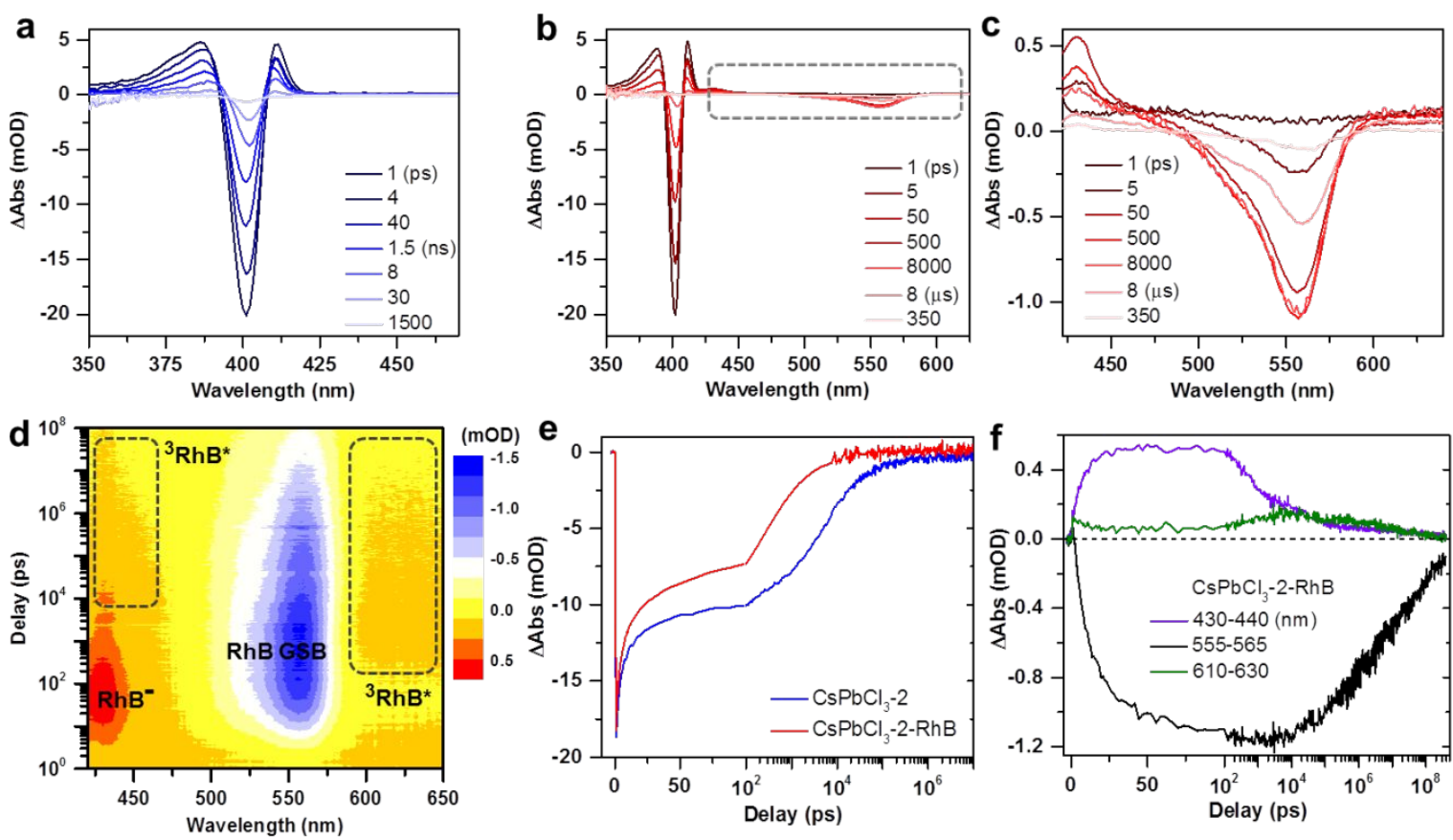

Figure S5. TA results for $\mathrm{CsPbCl}_{3}-2$. (a,b) TA spectra of (a) $\mathrm{CsPbCl}_{3}-2 \mathrm{NCs}$ and (b) $\mathrm{NC}-\mathrm{RhB}$ complexes probed at indicated time delays following $330 \mathrm{~nm}$ excitation. (c) The enlarged TA spectra in the spectral features of RhB (dashed in panel b). (d) 2D pseudocolor TA spectrum of NC-RhB complexes zooming in the spectral features of RhB (dashed in panel b). The GSB of $\mathrm{RhB}$ and absorptions of $\mathrm{RhB}^{-}$and ${ }^{3} \mathrm{RhB} *$ are indicated. (e) TA kinetics of $\mathrm{CsPbCl}_{3}-2 \mathrm{NCs}$ and NC-RhB complexes probed at XB wavelength (399-403 nm). (f) TA kinetics of NC-RhB complexes probed at the GSB (555-565 nm, balck), $\mathrm{RhB}^{-}(430-440 \mathrm{~nm}$, violet; also contains $\left.{ }^{3} \mathrm{RhB}^{*}\right)$ and ${ }^{3} \mathrm{RhB} *(610-630 \mathrm{~nm}$, green $)$. 

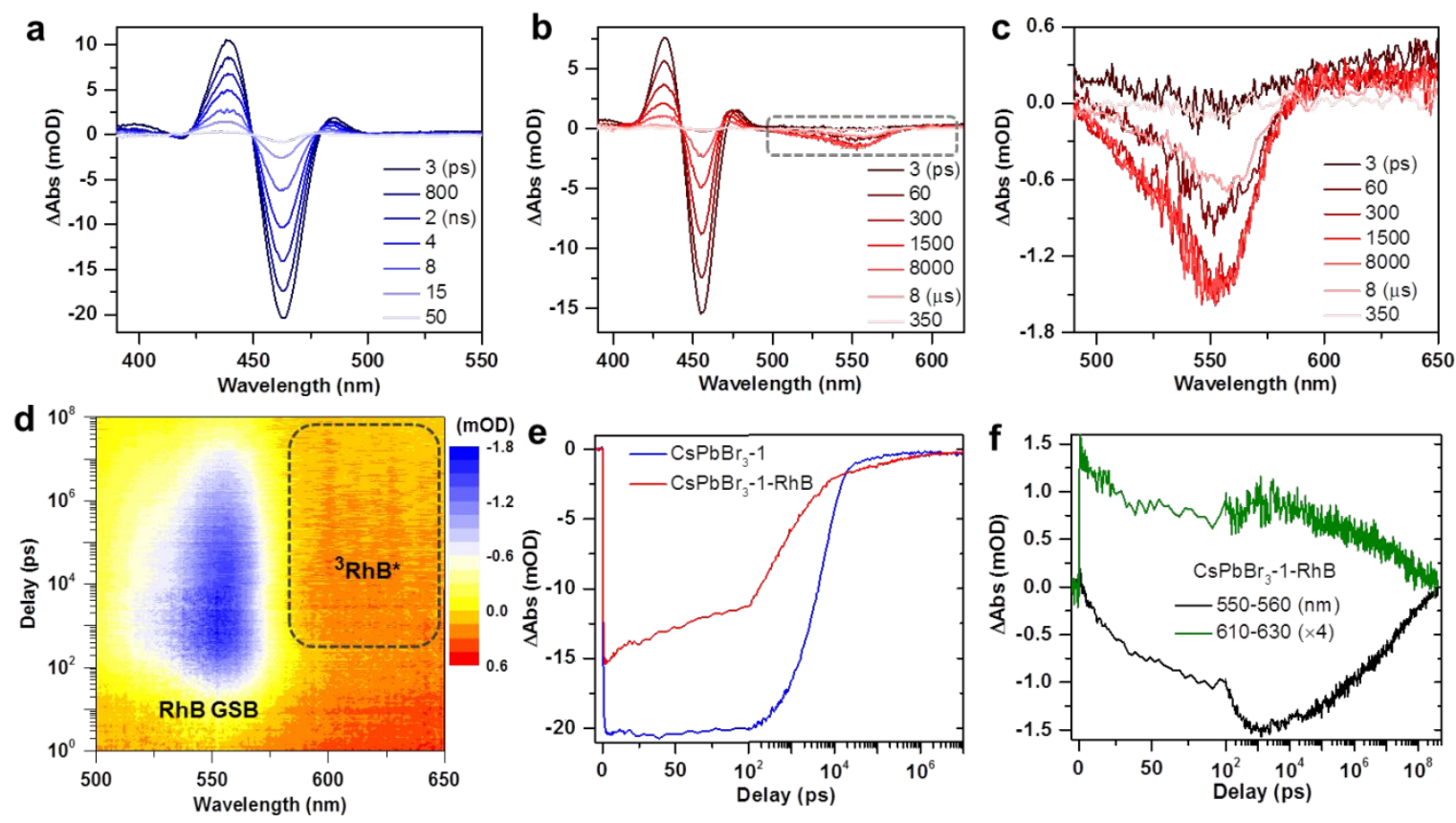

Figure S6. TA results for $\mathrm{CsPbBr}_{3}-1$. (a,b) TA spectra of (a) $\mathrm{CsPbBr}_{3}-1$ NCs and (b) NC-RhB complexes probed at indicated time delays following $330 \mathrm{~nm}$ excitation. (c) The enlarged TA spectra in the spectral features of $\mathrm{RhB}$ (dashed in panel b). (d) 2D pseudocolor TA spectrum of NC-RhB complexes zooming in the spectral features of RhB (dashed in panel b). The GSB of $\mathrm{RhB}$ and absorptions of ${ }^{3} \mathrm{RhB}^{*}$ are indicated. (e) TA kinetics of $\mathrm{CsPbBr}_{3}-1$ NCs and NC-RhB complexes probed at XB peaks. (f) TA kinetics of NC-RhB complexes probed at the GSB $\left(550-560 \mathrm{~nm}\right.$, balck) and ${ }^{3} \mathrm{RhB} *(610-630 \mathrm{~nm}$, green). The kinetics of ${ }^{3} \mathrm{RhB} *$ is amplified by a factor of 4 for clear comparison. 

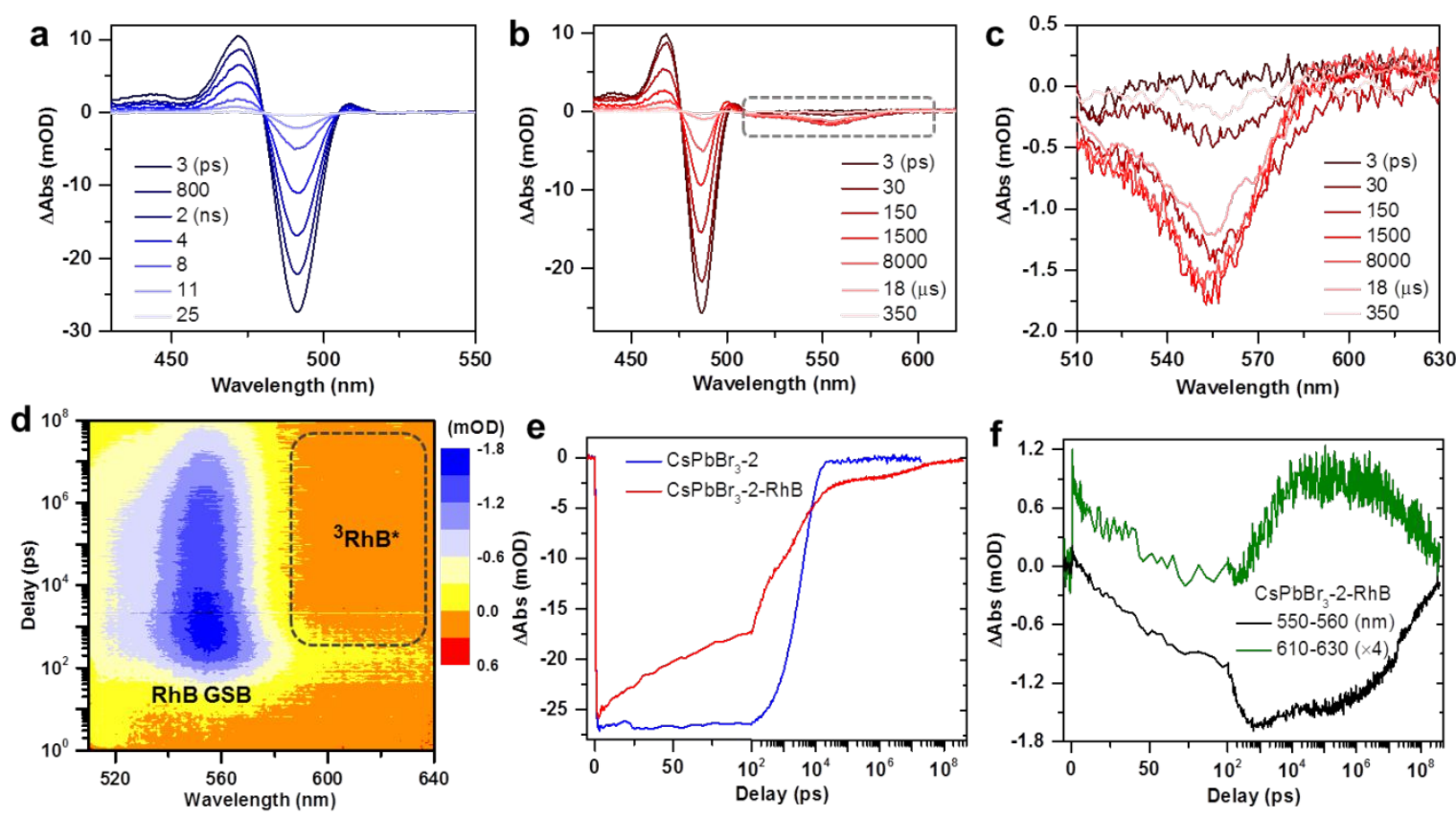

Figure S7. TA results for $\mathrm{CsPbBr}_{3}-2$. (a,b) TA spectra of (a) $\mathrm{CsPbBr}_{3}-2 \mathrm{NCs}$ and (b)

NC-RhB complexes probed at indicated time delays following $330 \mathrm{~nm}$ excitation. (c) The enlarged TA spectra in the spectral features of $\mathrm{RhB}$ (dashed in panel b). (d) 2D pseudocolor TA spectrum of NC-RhB complexes zooming in the spectral features of RhB (dashed in panel b). The GSB of $\mathrm{RhB}$ and absorptions of ${ }^{3} \mathrm{RhB}^{*}$ are indicated. (e) TA kinetics of $\mathrm{CsPbBr}_{3}-2 \mathrm{NCs}$ and NC-RhB complexes probed at XB peaks. (f) TA kinetics of NC-RhB complexes probed at the GSB $\left(550-560 \mathrm{~nm}\right.$, balck) and ${ }^{3} \mathrm{RhB} *(610-630 \mathrm{~nm}$, green). The kinetics of ${ }^{3} \mathrm{RhB} *$ is amplified by a factor of 4 for clear comparison. 

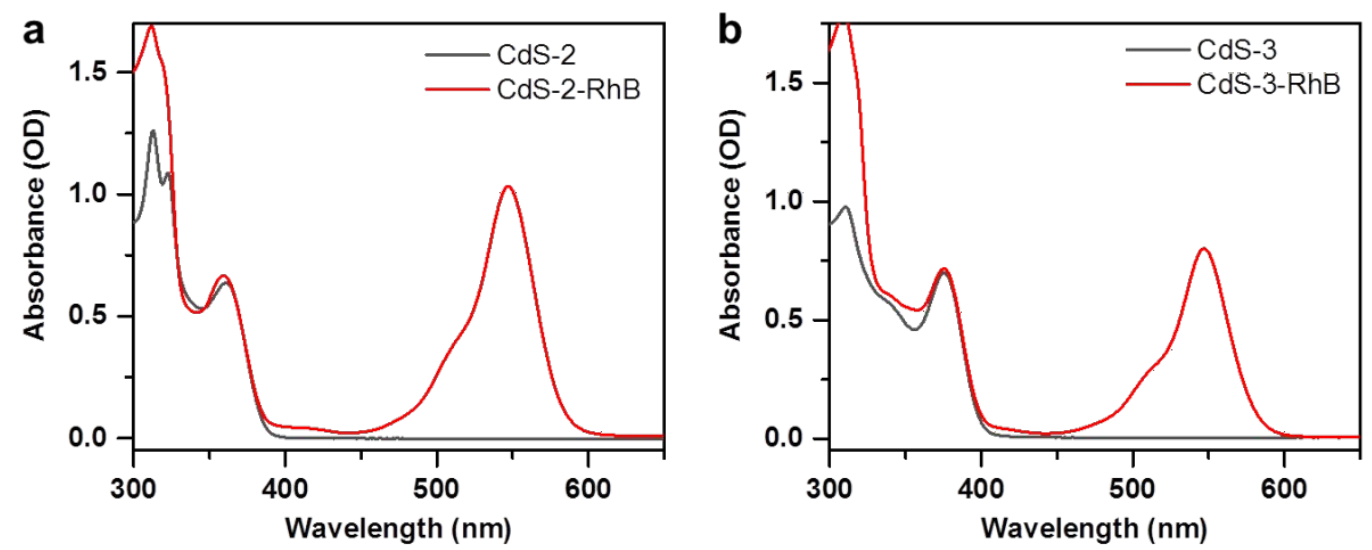

Figure S8. Absorption spectra of another two CdS NC samples, (a) CdS-2 and (b) CdS-3.
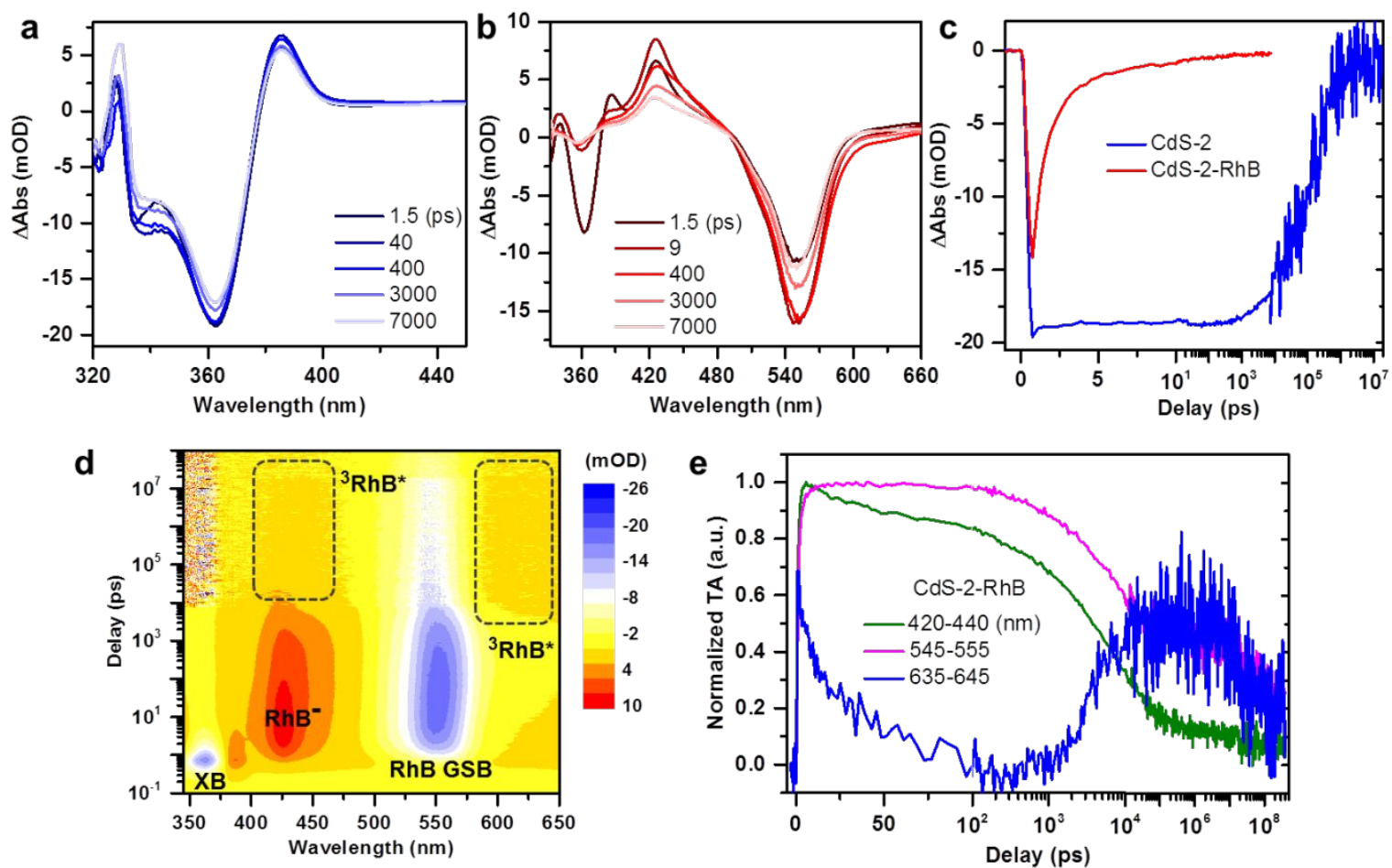

Figure S9. TA results for CdS-2. (a,b) TA spectra of (a) CdS-2 NCs and (b) NC-RhB complexes probed at indicated time delays following $330 \mathrm{~nm}$ excitation. (c) TA kinetics of CdS-2 NCs and NC-RhB complexes probed at XB peaks (362 nm). (d) 2D pseudocolor TA spectrum of NC-RhB complexes. The $\mathrm{XB}$ of $\mathrm{NC}, \mathrm{GSB}$ of $\mathrm{RhB}$ and absorptions of $\mathrm{RhB}^{-}$and 
${ }^{3} \mathrm{RhB}^{*}$ are indicated. (e) TA kinetics of NC-RhB complexes probed at the GSB (545-555 nm, magenta), $\mathrm{RhB}^{-}\left(420-440 \mathrm{~nm}\right.$, green; also contains $\left.{ }^{3} \mathrm{RhB}^{*}\right)$ and ${ }^{3} \mathrm{RhB} *(635-645 \mathrm{~nm}$, blue).
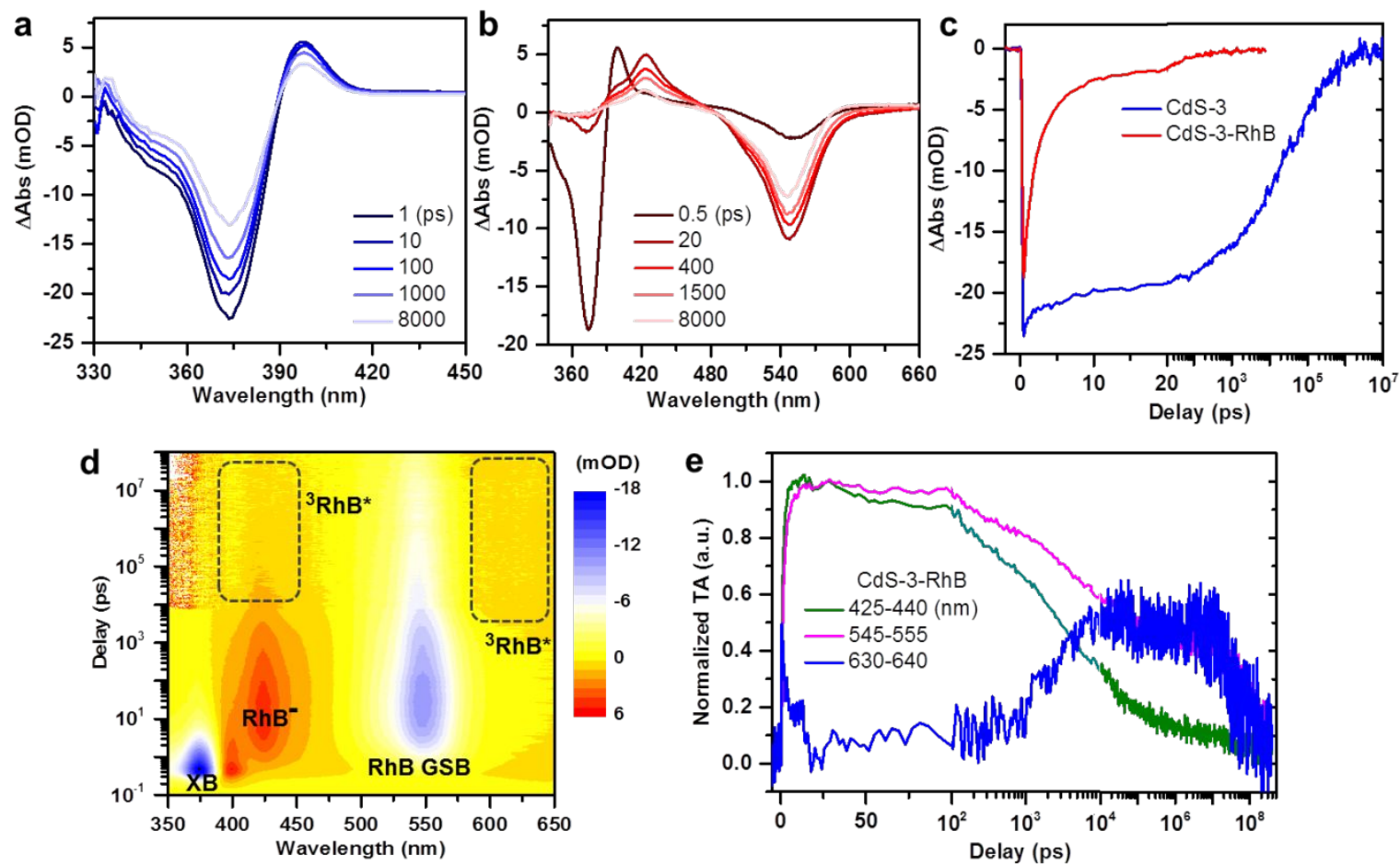

Figure S10. TA results for CdS-3. (a,b) TA spectra of (a) CdS-3 NCs and B) NC-RhB complexes probed at indicated time delays following $330 \mathrm{~nm}$ excitation. (c) TA kinetics of CdS-3 NCs and NC-RhB complexes probed at XB peaks $(375 \mathrm{~nm})$. (d) 2D pseudocolor TA spectrum of NC-RhB complexes. The XB of NC, GSB of RhB and absorptions of $\mathrm{RhB}^{-}$and ${ }^{3} \mathrm{RhB}^{*}$ are indicated. (e) TA kinetics of NC-RhB complexes probed at the GSB $(545-555 \mathrm{~nm}$, magenta), RhB- (425-440 nm, green; also contains $\left.{ }^{3} \mathrm{RhB}^{*}\right)$ and ${ }^{3} \mathrm{RhB}^{*}(630-640 \mathrm{~nm}$, blue). 


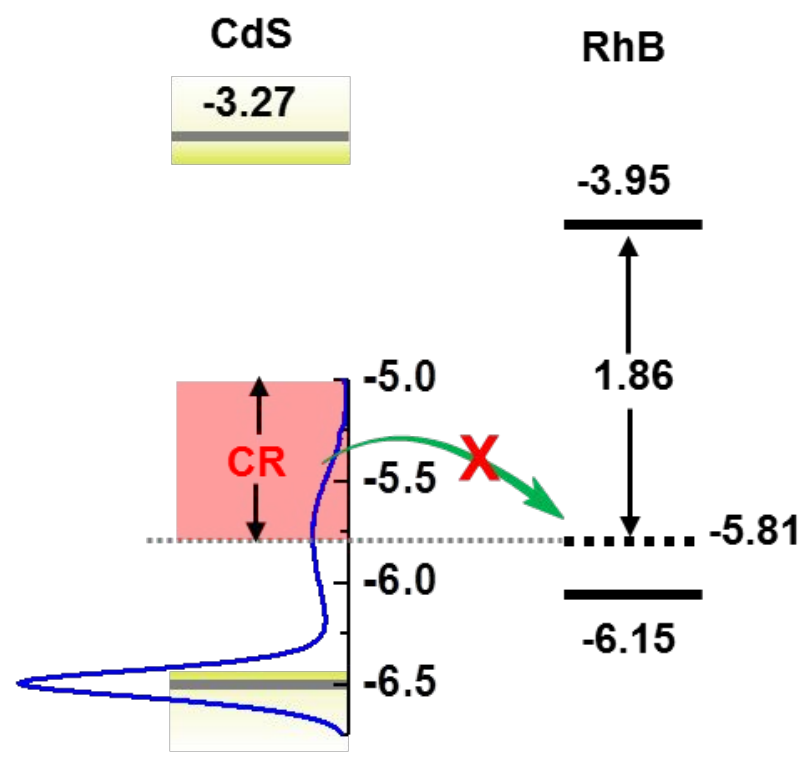

Figure S11. Energy level alignment in CdS NC-RhB, with the unit of eV vs vacuum. The triplet oxidation potential energy of $\mathrm{RhB}(-5.81 \mathrm{eV})$ is obtained by subtracting the triplet energy $(1.86 \mathrm{eV})$ from the LUMO. The PL spectrum of CdS NCs is used to derive the hole trap state energy distribution. This is done by aligning the band-edge emission peak center to the valence band edge $(-6.50 \mathrm{eV})$. Because the band-edge and trap-state emissions share the same conduction band energy level, the trap-state emission spectral shape directly reflects the hole trap state energy distribution. From the drawing, $-5.81 \mathrm{eV}$ is roughly at the peak position of the trap-state emission band, indicating that half of the trap states are below $-5.81 \mathrm{eV}$ while the other half is above $-5.81 \mathrm{eV}$. The latter does not have sufficient driving force for hole transfer from the trap states to $\mathrm{RhB}^{-}$for ${ }^{3} \mathrm{RhB}^{*}$ formation. Thus, the partition ratio between charge recombination and triplet formation pathways is $\sim 1: 1$. 

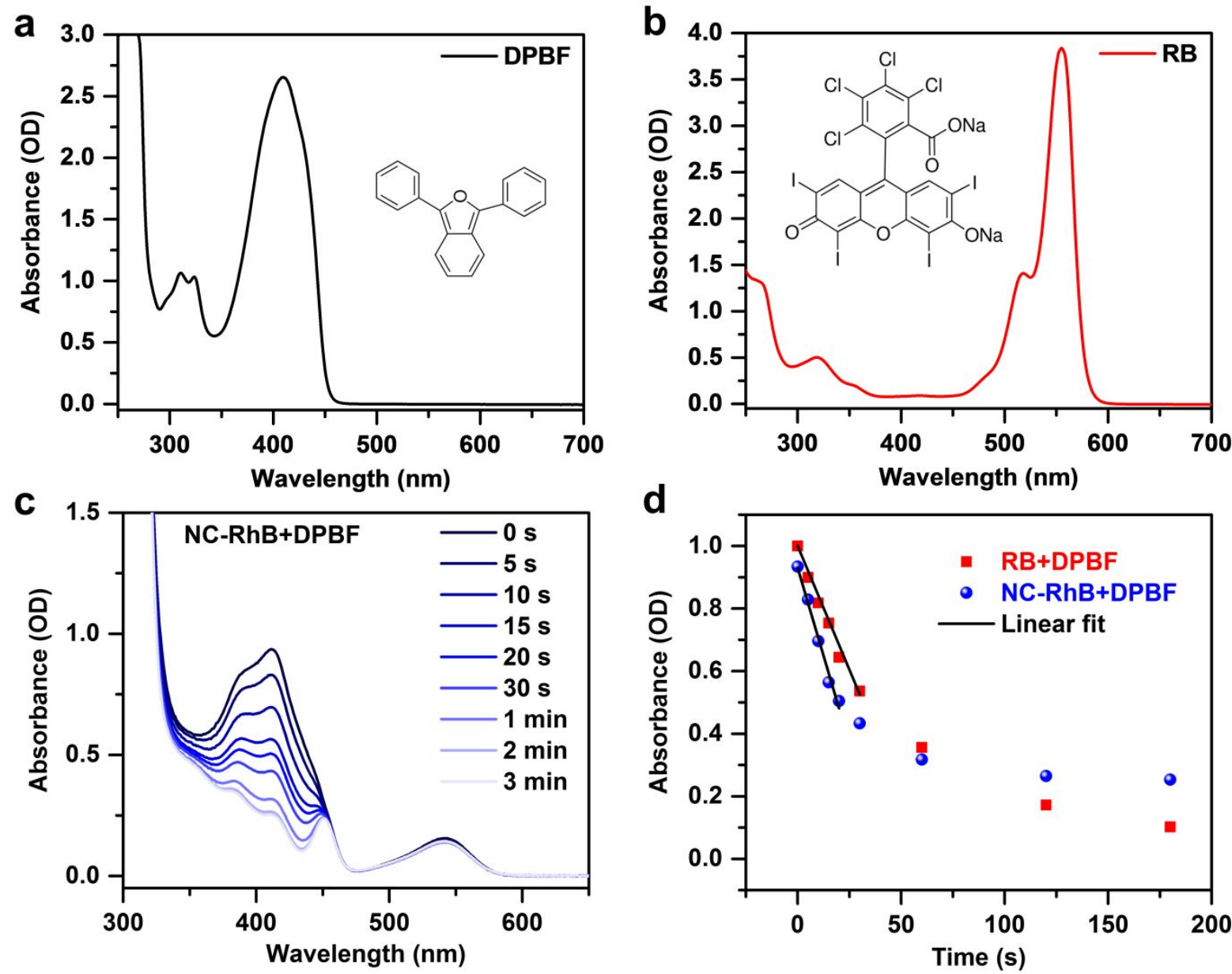

Figure S12. Singlet oxygen generation using $\mathrm{CsPbBr}_{3} \mathrm{NC}-\mathrm{RhB}$ complexes. (a,b) Absorption spectra and molecular structures of (a) 1,3-diphenylisobenzofuran (DPBF) and (b) Rose Bengal (RB) that are used as the ${ }^{1} \mathrm{O}_{2}$ scavenger and the standard sensitizer, respectively. (c) Representative time-dependent absorption spectra of $\mathrm{CsPbBr}_{3} \mathrm{NC}-1-\mathrm{RhB}$ complexes in the presence of DPBF in aerated hexane excited at $460 \mathrm{~nm}$. (d) Time-dependent absorbance of $\mathrm{DPBF}$ at $410 \mathrm{~nm}$ for $\mathrm{RB}$ (red squares) and $\mathrm{CsPbBr}{ }_{3} \mathrm{NC}-1-\mathrm{RhB}$ sensitizers. The black solid lines are linear fits to the data. 

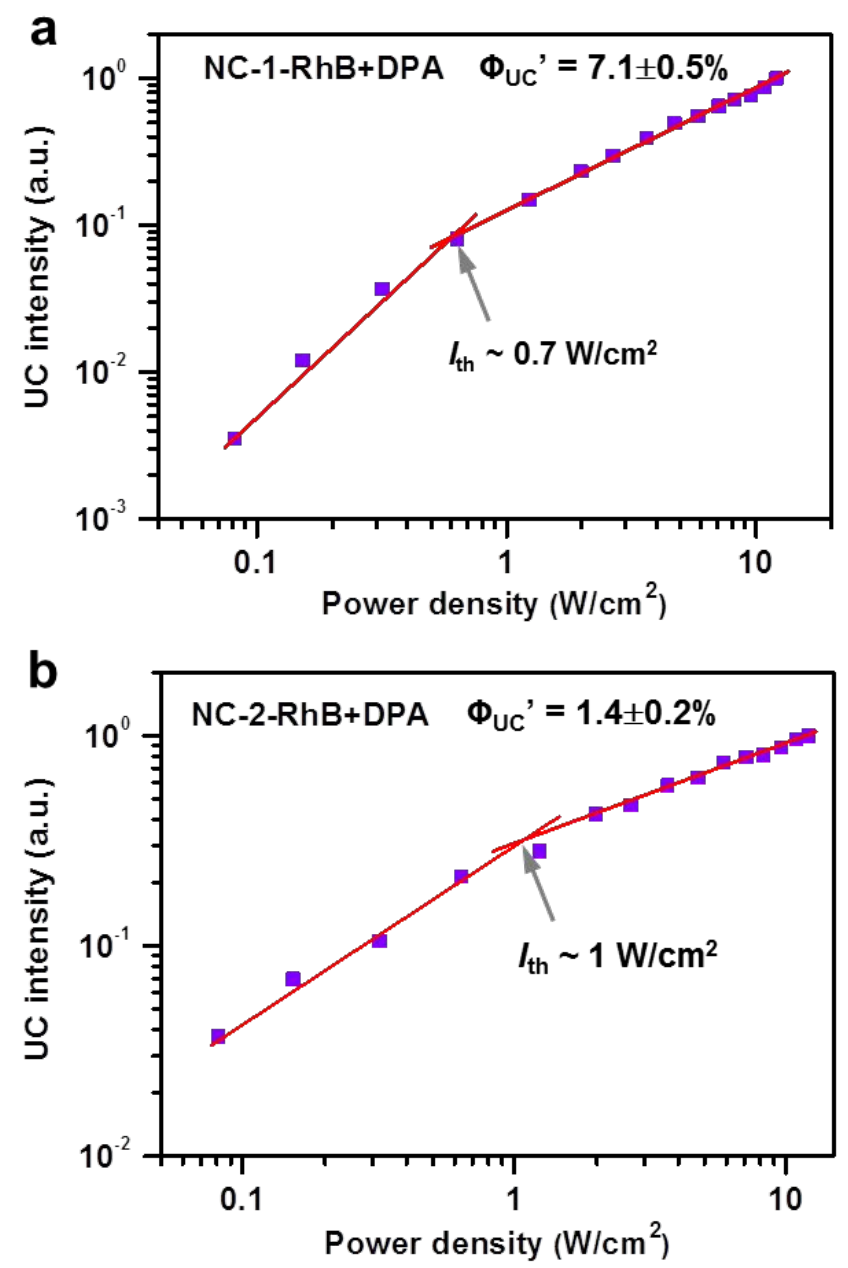

Figure S13. Integrated TTA-UC emission intensities as a function of the excitation power density for (a) NC-1-RhB and (b) NC-2-RhB samples. NC-1 and NC-2 refer to $\mathrm{CsPbBr}_{3} \mathrm{NCs}$ with their first absorption peaks at $\sim 460$ and $495 \mathrm{~nm}$, respectively. The violet squares are experimental data points. The red solid lines are their power fits which change from quadratic to linear dependences. The UC threshold $\left(I_{\mathrm{th}}\right)$ and quantum efficiency $\left(\Phi_{\mathrm{UC}}{ }^{\prime}\right)$ are labeled. 


\section{Experimental Methods}

Chemicals. The following reagents were used to prepare nanocrystal (NC)-molecule complexes: cesium carbonate $\left(\mathrm{Cs}_{2} \mathrm{CO}_{3}\right.$, Sigma-Aldrich, $\left.99.9 \%\right)$, lead(II) chloride $\left(\mathrm{PbCl}_{2}\right.$, Sigma-Aldrich, 99.999\%), lead(II) bromide ( $\mathrm{PbBr}_{2}$, Alfa Aesar, 98+\%), zinc bromide $\left(\mathrm{ZnBr}_{2}\right.$, Alfa Aesar, 99.9\%), cadmium oxide (CdO, Sigma-Aldrich, 99.99\%), oleic acid (OA, Sigma-Aldrich, 90\%), oleylamine (OAm, Acros Organics, 80-90\%), 1-octadecene (ODE, Sigma-Aldrich, 90\%), rhodamine B (RhB, Sigma-Aldrich, $\geqslant 95 \%)$, 9-anthracene carboxylic acid (ACA, Sigma-Aldrich, 99\%).

Synthesis of $\mathrm{CsPbCl}_{3}$ and $\mathrm{CsPbBr}_{3} \mathrm{NCs} \mathrm{CsPbCl}_{3}$ perovskite NCs were synthesized as described by Protesescu et $a l^{2}$. Briefly, $0.40 \mathrm{~g} \mathrm{Cs}_{2} \mathrm{CO}_{3}, 1.75 \mathrm{~mL} \mathrm{OA}$, and $15 \mathrm{~mL}$ ODE were degassed for 30 min under vacuum at $60{ }^{\circ} \mathrm{C}$, and heated under $\mathrm{Ar}$ atmosphere to $150{ }^{\circ} \mathrm{C}$ until all the $\mathrm{Cs}_{2} \mathrm{CO}_{3}$ powders were dissolved. This Cs-oleate precursor solution was kept at $100{ }^{\circ} \mathrm{C}$ for use. Then, $0.1 \mathrm{mmol}$ of $\mathrm{PbCl}_{2}, 5 \mathrm{~mL}$ ODE, $0.5 \mathrm{~mL} \mathrm{OA}$, and $0.5 \mathrm{~mL}$ OAm were loaded in a $25 \mathrm{~mL}$ three-neck flask and dried under vacuum for $1 \mathrm{~h}$ at $120{ }^{\circ} \mathrm{C}$. After degassing, the temperature was raised to $180{ }^{\circ} \mathrm{C}$ and $0.4 \mathrm{~mL}$ of previously synthesized Cs-oleate precursor was swiftly injected. 30 seconds after the injection, the NC solution was quickly cooled down to room temperature with an ice bath. The NCs were separated from the crude solution by centrifuging at $3000 \mathrm{rpm}$ for 30 mins. The precipitant was re-dispersed in $5 \mathrm{~mL}$ hexane by shaking and sonication, followed by centrifugation at $7830 \mathrm{rpm}$ for $2 \mathrm{mins}$ in order to remove larger NCs and agglomerates.

$\mathrm{CsPbBr}_{3}$ perovskite NCs were synthesized by using a hot injection approach with modifications ${ }^{3}$. The synthesis started with preparation of Cs-oleate precursors $\left(0.25 \mathrm{~g} \mathrm{Cs}_{2} \mathrm{CO}_{3}\right.$ 
was degassed in $0.8 \mathrm{~g} \mathrm{OA}$ and $7 \mathrm{~g} \mathrm{ODE}$ at $150{ }^{\circ} \mathrm{C}$ for $1 \mathrm{~h}$, and then kept at $100{ }^{\circ} \mathrm{C}$ for use). In another $250 \mathrm{~mL}$ 3-neck flask, the precursor solution of $\mathrm{Pb}$ and $\mathrm{Br}$ was prepared by dissolving $0.45 \mathrm{~g} \mathrm{PbBr}_{2}$ and $1.10 \mathrm{~g} \mathrm{ZnBr}_{2}$ in a mixture of $\mathrm{OA}(16 \mathrm{~mL}), \mathrm{OAm}(16 \mathrm{~mL})$ and $\mathrm{ODE}(26$ $\mathrm{mL}$ ). Then, the precursor solution was vacuum-dried for $1 \mathrm{~h}$ at $110^{\circ} \mathrm{C}$, and heated under $\mathrm{Ar}$ atmosphere to $140{ }^{\circ} \mathrm{C}$ until all the powders were dissolved. After the solution was cooled and kept at $110{ }^{\circ} \mathrm{C}, 2.4 \mathrm{~mL}$ of Cs-oleate precursor was injected to initiate the reaction. The reaction was quenched after 6 mins by cooling the flask in an ice bath. The product was centrifuged at $4000 \mathrm{rpm}$ for $15 \mathrm{~min}$ to remove the unreacted salts as the precipitate, and the perovskite NCs dispersed in the supernatant were collected. $10 \mathrm{~mL}$ of acetone was directly added into the supernatant to precipitate the NCs followed by centrifuging at $4000 \mathrm{rpm}$ for 5 mins. The dried NCs were collected and dissolved in hexane. The lowest energy absorption peak of as-prepared $\mathrm{CsPbBr}_{3} \mathrm{NCs}$ is situated at $\sim 460 \mathrm{~nm}\left(\mathrm{CsPbBr}_{3}-1\right.$ sample). Another $\mathrm{CsPbBr}_{3} \mathrm{NCs}\left(\mathrm{CsPbBr}_{3}-2\right.$ sample) were obtained by tuning the reaction temperature and time (the lowest energy absorption peak at $\sim 490 \mathrm{~nm}$ ), as detailed in refs ${ }^{4,5}$.

Synthesis of CdS NCs. CdS NCs were synthesized according to a literature method ${ }^{6}$. Briefly, $0.077 \mathrm{~g} \mathrm{CdO}$, a varying amount of $\mathrm{OA}(1.8 \mathrm{~mL}, 1.2 \mathrm{~mL}$ and $1.5 \mathrm{~mL}$ for CdS, CdS-2 and CdS-3 samples, respectively) and $15 \mathrm{~mL}$ ODE were degassed under vacuum for $10 \mathrm{mins}$ at $90{ }^{\circ} \mathrm{C}$. The solution was then heated to $270{ }^{\circ} \mathrm{C}$ in 30 mins under Ar atmosphere until all the $\mathrm{CdO}$ powders were dissolved. Then, $3 \mathrm{~mL}$ of sulfur $(\mathrm{S})$ stock solution (0.1 $\mathrm{M}$ of $\mathrm{S}$ in $\mathrm{ODE})$ was swiftly injected. The reaction was stopped after $30 \mathrm{~s}$ by injecting $7 \mathrm{~mL}$ room-temperature ODE and removing the heating mantle. The CdS NCs were purified several times at 7800 rpm for 5 mins with ethanol and finally dispersed in hexane for use. 
Preparation of NC-RhB complexes. The NC-RhB complexes were prepared by adding RhB powders into a NC solution in hexane, followed by sonication for controlled time (5-30 mins). The mixture was filtered to obtain a clear solution containing $\mathrm{NC}-\mathrm{RhB}$ complexes; because the solubility of $\mathrm{RhB}$ in hexane is negligible, all the $\mathrm{RhB}$ molecules were believed to be anchored to NC surfaces. On the basis of the molecular structure of RhB (Fig. S2d), there should be a large steric hindrance for the carboxyl group to access NC surface. Nonetheless, we believe the carboxyl group still plays a key role in anchoring the molecule on to NC surface. This is because we found it very difficult to attach another molecule with a similar ammonium inner salt structure but no carboxyl group, methylene blue, onto the surface of perovskite NCs.

On the basis of the extinction coefficients of $\mathrm{CsPbCl}_{3} \mathrm{NCs}\left(\varepsilon_{400 \mathrm{~nm}}=6.3 \times 10^{5} \mathrm{M}^{-1} \mathrm{~cm}^{-1}\right)^{7}, \mathrm{CdS}$ NCs $\left(\varepsilon_{382 \mathrm{~nm}}=2.5 \times 10^{5} \mathrm{M}^{-1} \mathrm{~cm}^{-1}\right)^{8}$ and RhB molecule $\left(\varepsilon_{543 \mathrm{~nm}}=106000 \mathrm{M}^{-1} \mathrm{~cm}^{-1}\right)^{9}$, on average 3 and 3.5 molecules were bound to $\mathrm{CsPCl}_{3}$ and $\mathrm{CdS} \mathrm{NCs}$, respectively.

TEM measurements. TEM images were acquired on a JEOL JEM-2100 TEM at $200 \mathrm{KV}$ (line resolution of $0.14 \mathrm{~nm}$ ) by drop casting the $\mathrm{NC}$-hexane dispersions on carbon-coated $\mathrm{Cu}$ grids and drying under ambient conditions.

Transient absorption (TA). The femtosecond pump-probe TA measurements were performed using a regenerative amplified Ti:sapphire laser system (Coherent; $800 \mathrm{~nm}, 70 \mathrm{fs}$, $6 \mathrm{~mJ} /$ pulse, and $1 \mathrm{kHz}$ repetition rate) as the laser source and a HELIOS Fire spectrometer (Ultrafast Systems LLC). Briefly, the $800 \mathrm{~nm}$ output pulse from the regenerative amplifier was split in two parts with a $50 \%$ beam splitter. The transmitted part was used to pump a TOPAS Optical Parametric Amplifier (OPA) which generated a wavelength-tunable laser 
pulse from $250 \mathrm{~nm}$ to $2.5 \mu \mathrm{m}$ as pump beam. The reflected $800 \mathrm{~nm}$ beam was split again into two parts. One part with less than $10 \%$ was attenuated with a neutral density filter and focused into a crystal to generate a white light continuum (WLC) used for probe beam. The probe beam was focused with an Al parabolic reflector onto the sample. After the sample, the probe beam was collimated and then focused into a fiber-coupled spectrometer with CMOS sensors and detected at a frequency of $1 \mathrm{KHz}$. The intensity of the pump pulse used in the experiment was controlled by a variable neutral-density filter wheel. The delay between the pump and probe pulses was controlled by a motorized delay stage. The pump pulses were chopped by a synchronized chopper at $500 \mathrm{~Hz}$ and the absorbance change was calculated with two adjacent probe pulses (pump-blocked and pump-unblocked). The samples were placed in $1 \mathrm{~mm}$ airtight cuvettes in a $\mathrm{N}_{2}$-filled glove box and measured under ambient conditions. Nanosecond TA was performed with the EOS spectrometer (Ultrafast Systems LLC). The pump beam is generated in the same way as the femtosecond TA experiment described above. A different white light continuum $(380-1700 \mathrm{~nm}, 0.5 \mathrm{~ns}$ pulse width, $20 \mathrm{kHz}$ repetition rate) was used, which was generated by focusing a Nd:YAG laser into a photonic crystal fiber. The delay time between the pump and probe beam was controlled by a digital delay generator (CNT-90, Pendulum Instruments).

Time-resolved PL. For an 8 ns time window, the PL decay was measured using a time-resolved fluorescence upconversion set-up (Chimera, Light conversion) and a Pharos laser $(1030 \mathrm{~nm}, 100 \mathrm{kHz}, 230 \mathrm{fs}$ pulse-duration; Light conversion). Briefly, the fundamental $1030 \mathrm{~nm}$ laser pulse was split into two parts. One part was used to pump a TOPAS OPA to generate wavelength-tunable excitation pulses; the other was used as the gate pulse. The 
emitted light was collected by lens and focused into a BBO crystal together with the $1030 \mathrm{~nm}$ gate pulse to generate the up-converted signal via sum-frequency-generation (SFG). The up-converted photons were focused onto the entrance slit of a monochromator and then detected by the spectrometer. The fluorescence decay curve was obtained by delaying the gate pulse using a mechanical delay stage. For a 100 ns time window, the time-resolved PL decay was measured using time-correlated single-photon counting (TCSPC) set-up, which uses the same excitation source and camera as the fluorescence upconversion set-up and has a temporal resolution of 100 ps. All samples were placed in $1 \mathrm{~mm}$ airtight cuvettes in a $\mathrm{N}_{2}$-filled glove box and were vigorously stirred in all the measurements.

Singlet Oxygen generation. The singlet oxygen $\left({ }^{1} \mathrm{O}_{2}\right)$ generation experiments were performed for air equilibrated samples. In brief, $\mathrm{CsPbBr}_{3} \mathrm{NC}-\mathrm{RhB}$ complexes were mixed with 1,3-diphenylisobenzofuran (DPBF) in hexane. DPBF was used as ${ }^{1} \mathrm{O}_{2}$ scavenger because it can trap the generated ${ }^{1} \mathrm{O}_{2}$ through oxidation ${ }^{10}$. The as-prepared samples were irradiated with a $460 \mathrm{~nm}$ laser light $(30 \mathrm{~mW}$; from the Pharos laser) with variable excitation time. The ${ }^{1} \mathrm{O}_{2}$ production sensitized by $\mathrm{NC}-\mathrm{RhB}$ was monitored by following the absorbance of DPBF at $410 \mathrm{~nm}$. We calculated ${ }^{1} \mathrm{O}_{2}$ quantum yield $\left(\Phi_{\Delta}\right)$ using a relative method with Rose Bengal (RB) as the standard sensitizer (sensitization quantum yield $\Phi_{\text {std }}=80 \%$ in methanol) ${ }^{11}$. The $\Phi_{\Delta}$ of the NC-RhB sensitizer was calculated according to the following equation ${ }^{12}$ : $\Phi_{\Delta}=\Phi_{\text {std }} \times\left(\frac{N_{\text {std }}}{N_{\text {sam }}} \times \frac{1-10^{-A_{\text {std }}}}{1-10^{-A_{\text {sam }}}}\right) \times\left(\frac{m_{\text {sam }}}{m_{\text {std }}}\right) \times\left(\frac{n_{\text {sam }}}{n_{\text {std }}}\right)^{2}$

where "sam" and "std" represent the sample and the standard. $\Phi, N, A, m$, and $n$ are the singlet oxygen quantum yield, the total number of incident photons, the absorbance at excitation wavelength, the slope of the absorbance of DPBF changing over time, and the 
refractive index of the solvent used for measurement, respectively.

Triplet-triplet annihilation photon upconversion (TTA-UC). TTA-UC measurements were performed with a $447 \mathrm{~nm} c$ w laser under deaerated conditions. In brief, $\mathrm{CsPbBr}_{3}$ NC-RhB complexes were mixed with diphenyl anthracene (DPA) in hexane. The as-prepared samples were irradiated with $c w$ laser light with variable excitation intensity. The laser beam was focused by a quartz lens. The focal point was at the air-free cuvettes with the samples. Along the axis perpendicular to the excitation laser, a lens system was used to collect the fluorescence and couple it into a fiber. The other side of the fiber was connected to a spectrometer (Maya2000 pro; Ocean Optics). The upconversion quantum yield $\left(\Phi_{\mathrm{UC}}{ }^{\prime}\right)$ was calculated using Coumarin 503 as a reference with quantum yield of 0.75 in ethanol. The $\Phi_{\mathrm{UC}}$ ' was calculated according to the following equation:

$$
\Phi_{\mathrm{UC}}{ }^{\prime}=2 \times \Phi_{\mathrm{std}} \times\left(\frac{1-10^{-A_{\text {std }}}}{1-10^{-A_{\text {sam }}}}\right) \times\left(\frac{F_{\text {sam }}}{F_{\text {std }}}\right) \times\left(\frac{n_{\text {sam }}}{n_{\text {std }}}\right)^{2}
$$

where "sam" and "std" represent the sample and the standard. $\Phi, A, F$, and $n$ are the photoluminescence quantum yield, the absorbance at the excitation wavelength, the integrated photon number, and the refractive index of the solvent used for measurement, respectively. 


\section{Kinetics fitting}

Model for $\mathrm{CsPbCl}_{3}$ NC-RhB complexes. According to the TA and PL measurements, a kinetic fitting model is proposed as follows: upon selective photoexcitation $\mathrm{CsPbCl}_{3} \mathrm{NCs}$ in $\mathrm{NC}-\mathrm{RhB}$ complexes, the band-edge electrons transfer (ET) from $\mathrm{NC}$ to $\mathrm{RhB}$ within $\sim 20$ ps to form charge separated states $\left(\mathrm{NC}^{+}-\mathrm{RhB}^{-}\right)$with a rate of $k_{\mathrm{ET}}$ (Stage I). From $\sim 20$ ps to $10 \mathrm{~ns}$ timescale, the band-edge holes in $\mathrm{NC}^{+}$are injected into the $\mathrm{RhB}^{-}$to form $\mathrm{NC}^{-}{ }^{3} \mathrm{RhB}^{*}$ with a rate of $k_{\mathrm{HT}}$, while the absorptive signal of ${ }^{3} \mathrm{RhB}^{*}$ grows in and the ground state bleach (GSB) signal of RhB maintains constant (Stage II). From 10 ns to $\mu$ s timescale, the generated triplets $\left({ }^{3} \mathrm{RhB}^{*}\right)$ decay out to form to NC-RhB (Stage III).

The formation kinetics of $\mathrm{RhB}$ GSB and $\mathrm{RhB}^{-}$are simultaneously fitted using a mono-exponential decay function $\left(S(t) \propto A \cdot e^{-\left(k_{E T}+k_{E t r}\right) t}\right)$. Here $k_{\mathrm{Etr}}$ is the rate constant for the electron trapping process that competes with electron transfer, which is $\sim 0.045 \mathrm{ps}^{-1}$ from the kinetics of free $\mathrm{CsPbCl}_{3} \mathrm{NCs}$. The ensuing hole transfer and ${ }^{3} \mathrm{RhB}^{*}$ formation kinetics are simultaneously fitted using two-exponential decay functions $\left(S(t) \propto A_{1} \cdot e^{-k_{1} t}+A_{2} \cdot e^{-k_{2} t}\right)$, where we have assumed that the hole in the charge separated state has a much longer intrinsic lifetime than the hole transfer process. Lastly, the ${ }^{3} \mathrm{RhB} *$ decay is fitted using three-exponential decay functions $\left(S(t) \propto A_{1} \cdot e^{-k_{1} t}+A_{2} \cdot e^{-k_{2} t}+A_{3} \cdot e^{-k_{3} t}\right)$. Note that the criterion for the choice of the number of exponential components is to use the fewest components while ensuring fitting convergence. The fitting parameters are listed in Table S1, and the rate constants are converted to time constants in the table. The average lifetime $\tau_{\text {ave }}$ was calculated according to the following equation: 


$$
\tau_{\text {ave }}=\frac{\sum_{i} A_{i} \tau_{i}}{\sum_{i} A_{i}}
$$

where $A_{\mathrm{i}}$ and $\tau_{\mathrm{i}}$ are the amplitude and lifetime, respectively, of the $i$ th exponential component.

Table S1. Fitting parameters for $\mathrm{CsPbCl}_{3} \mathrm{NC}-\mathrm{RhB}$ complexes.

\begin{tabular}{|c|c|c|c|c|c|c|c|c|}
\hline \multirow{2}{*}{$\begin{array}{c}\text { Probe } \\
\text { wavelength } \\
(\mathrm{nm})\end{array}$} & Electron & \multicolumn{3}{|c|}{ Hole transfer } & \multicolumn{4}{|c|}{ Triplet lifetime } \\
\hline & $\begin{array}{l}\tau_{\mathrm{ET}}(\mathrm{ps}) \\
\mathrm{A}(\%)\end{array}$ & $\begin{array}{l}\tau_{1}(\mathrm{ps}) \\
\mathrm{A}_{1}(\%)\end{array}$ & $\begin{array}{l}\tau_{2}(\mathrm{ps}) \\
\mathrm{A}_{2}(\%)\end{array}$ & $\begin{array}{c}\tau_{\mathrm{ave}, \mathrm{HT}} \\
(\mathrm{ps})\end{array}$ & $\begin{array}{l}\tau_{1}(\mu \mathrm{s}) \\
\mathrm{A}_{1}(\%)\end{array}$ & $\begin{array}{l}\tau_{2}(\mu \mathrm{s}) \\
\mathrm{A}_{2}(\%)\end{array}$ & $\begin{array}{l}\tau_{3}(\mu \mathrm{s}) \\
\mathrm{A}_{3}(\%)\end{array}$ & $\begin{array}{l}\tau_{\mathrm{ave}, \mathrm{T}} \\
(\mu \mathrm{s})\end{array}$ \\
\hline $\begin{array}{l}430-440 \\
\left(\mathrm{RhB}^{-+}\right. \\
\left.{ }^{3} \mathrm{RhB}^{*}\right)\end{array}$ & $\begin{array}{c}6.6 \pm 0.3 \\
(100)\end{array}$ & $\begin{array}{c}359 \pm 27 \\
(69)\end{array}$ & $\begin{array}{c}8856 \pm 1056 \\
(31)\end{array}$ & $2993 \pm 346$ & $\begin{array}{c}0.54 \pm 0.03 \\
(44)\end{array}$ & $\begin{array}{c}16.5 \pm 2.1 \\
(31)\end{array}$ & $\begin{array}{c}198 \pm 23.3 \\
(25)\end{array}$ & $54.9 \pm 6.5$ \\
\hline $\begin{array}{r}555-565 \\
(\mathrm{GSB})\end{array}$ & $\begin{array}{c}6.6 \pm 0.3 \\
(100)\end{array}$ & - & - & - & $\begin{array}{c}0.54 \pm 0.03 \\
(44)\end{array}$ & $\begin{array}{c}16.5 \pm 2.1 \\
(31)\end{array}$ & $\begin{array}{c}198 \pm 23.3 \\
(25)\end{array}$ & $54.9 \pm 6.5$ \\
\hline $\begin{array}{l}610-630 \\
\left({ }^{3} \mathrm{RhB}^{*}\right)\end{array}$ & - & $\begin{array}{c}359 \pm 27 \\
(69)\end{array}$ & $\begin{array}{c}8856 \pm 1056 \\
\text { (31) }\end{array}$ & $2993 \pm 346$ & $\begin{array}{c}0.54 \pm 0.03 \\
(44)\end{array}$ & $\begin{array}{c}16.5 \pm 2.1 \\
(31)\end{array}$ & $\begin{array}{c}198 \pm 23.3 \\
(25)\end{array}$ & $54.9 \pm 6.5$ \\
\hline
\end{tabular}

Model for CdS NC-RhB complexes. According to the TA and PL measurements, a kinetic fitting model is proposed as follows: upon selective photoexcitation CdS NCs in NC-RhB complexes, the band-edge electrons quickly transfer from $\mathrm{NC}$ to $\mathrm{RhB}$ within $\sim 10 \mathrm{ps}$ to form $\mathrm{RhB}^{-}$with a rate of $k_{\mathrm{ET}}$, while a sub-ensemble of band-edge holes are quickly trapped in several picoseconds through ultrafast hole trapping (HTr). In addition, the RhB GSB and 
absorptive signal of $\mathrm{RhB}^{-}$grow in a simultaneous way. From $\sim 10$ ps to 10 ns timescale, a fraction of $\mathrm{RhB}^{-}$decay via charge recombination $(\mathrm{CR})$ to form ground state $\mathrm{NC}-\mathrm{RhB}$, along with a simultaneous decay of RhB GSB signal with a rate of $k_{\mathrm{CR}}$. On the other hand, a part of trapped holes are injected into the rest $\mathrm{RhB}^{-}$to form $\mathrm{NC}^{-}{ }^{3} \mathrm{RhB}^{*}$ with a rate of $k_{\mathrm{HT}}$, while the absorptive signal of ${ }^{3} \mathrm{RhB}^{*}$ grows in. From $\sim 10 \mathrm{~ns}$ to $\mu$ s timescale, the generated triplets $\left({ }^{3} \mathrm{RhB} *\right)$ decay out to form to NC-RhB.

The formation kinetics of RhB GSB are fitted using a mono-exponential decay function $\left(S(t) \propto A \cdot e^{-k_{E T} t}\right)$. The intrinsic decay rate of electrons is ignored in this equation because band edge electrons in CdS are long-lived. The ensuing hole transfer kinetics are fitted using a mono-exponential decay function $\left(S(t) \propto A \cdot e^{-k_{H T} t}\right)$, and charge recombination kinetics are fitted using two-exponential decay functions $\left(S(t) \propto A_{1} \cdot e^{-k_{1} t}+A_{2} \cdot e^{-k_{2} t}\right)$. Lastly, the ${ }^{3} \mathrm{RhB} *$ decay is fitted using another two-exponential decay functions $\left(S(t) \propto A_{1} \cdot e^{-k_{1} t}+A_{2} \cdot e^{-k_{2} t}\right)$. Again, the criterion for the choice of the number of exponential components is to use the fewest components while ensuring fitting convergence. The fitting parameters are listed in Table S2, and the rate constants are converted to time constants in the table. The average lifetime $\tau_{\mathrm{ave}}$ was calculated according to the equation S3.

Table S2. Fitting parameters for CdS NC-RhB complexes.

\begin{tabular}{|c|c|c|c|c|c|c|c|c|}
\hline \multirow{2}{*}{ Probe } & Electron & Hole & \multicolumn{2}{|c|}{ Charge recombination } & \multicolumn{2}{|c|}{ Triplet lifetime } \\
$(\mathrm{nm})$ & $\tau_{\mathrm{ET}}(\mathrm{ps})$ & $\tau_{\mathrm{HT}}(\mathrm{ps})$ & $\tau_{1}(\mathrm{ps})$ & $\tau_{2}(\mathrm{ps})$ & $\tau_{\mathrm{ave}, \mathrm{CR}}$ & $\tau_{1}(\mu \mathrm{s})$ & $\tau_{2}(\mu \mathrm{s})$ & $\tau_{\mathrm{ave}, \mathrm{T}}(\mu \mathrm{s})$ \\
& $\mathrm{A}(\%)$ & $\mathrm{A}(\%)$ & $\mathrm{A}_{1}(\%)$ & $\mathrm{A}_{2}(\%)$ & $(\mathrm{ps})$ & $\mathrm{A}_{1}(\%)$ & $\mathrm{A}_{2}(\%)$ & \\
\cline { 2 - 9 } & transfer & transfer & \multicolumn{2}{|c|}{$(\%)$} & \\
\hline
\end{tabular}




\begin{tabular}{|c|c|c|c|c|c|c|c|c|}
\hline $550-560$ & $1.4 \pm 0.1$ & - & $707 \pm 52$ & $10064 \pm 304$ & $6976 \pm 221$ & $7.2 \pm 0.7$ & $182 \pm 7$ & $128 \pm 5.0$ \\
$(\mathrm{GSB})$ & $(100)$ & & $(33)$ & $(67)$ & & $(31)$ & $(69)$ & \\
\hline $630-640$ & - & $2955 \pm 520$ & - & - & - & $7.2 \pm 0.7$ & $182 \pm 7$ & $128 \pm 5.0$ \\
$\left({ }^{3} \mathrm{RhB}^{*}\right)$ & & $(100)$ & & & & $(31)$ & $(69)$ & \\
\hline
\end{tabular}

\section{References for SI.}

(1) Luo, X.; Lai, R.; Li, Y.; Han, Y.; Liang, G.; Liu, X.; Ding, T.; Wang, J.; Wu, K. Triplet Energy Transfer from $\mathrm{CsPbBr} 3$ Nanocrystals Enabled by Quantum Confinement. $J$. Am. Chem. Soc. 2019, 141, 4186-4190.

(2) Protesescu, L.; Yakunin, S.; Bodnarchuk, M. I.; Krieg, F.; Caputo, R.; Hendon, C. H.; Yang, R. X.; Walsh, A.; Kovalenko, M. V. Nanocrystals of cesium lead halide perovskites $(\mathrm{CsPbX} 3, \mathrm{X}=\mathrm{Cl}, \mathrm{Br}$, and $\mathrm{I})$ : novel optoelectronic materials showing bright emission with wide color gamut. Nano letters 2015, 15, 3692-3696.

(3) Dong, Y.; Qiao, T.; Kim, D.; Parobek, D.; Rossi, D.; Son, D. H. Precise Control of Quantum Confinement in Cesium Lead Halide Perovskite Quantum Dots via Thermodynamic Equilibrium. Nano Lett. 2018, 18, 3716-3722.

(4) Li, Y.; Ding, T.; Luo, X.; Chen, Z.; Liu, X.; Lu, X.; Wu, K. Biexciton Auger recombination in mono-dispersed, quantum-confined $\mathrm{CsPbr} 3$ perovskite nanocrystals obeys universal volume-scaling. Nano Research 2019, 12, 619-623.

(5) Li, Y.; Lai, R.; Luo, X.; Liu, X.; Ding, T.; Lu, X.; Wu, K. On the absence of a phonon bottleneck in strongly confined $\mathrm{Cs} \mathrm{PbBr} 3$ perovskite nanocrystals. Chemical science 2019, 10, 5983-5989.

(6) Yu, W. W.; Peng, X. Formation of high - quality CdS and other II-VI semiconductor nanocrystals in noncoordinating solvents: tunable reactivity of monomers. Angewandte Chemie International Edition 2002, 41, 2368-2371.

(7) Ahumada-Lazo, R.; Alanis, J. A.; Parkinson, P.; Binks, D. J.; Hardman, S. J.; Griffiths, J. T.; Wisnivesky Rocca Rivarola, F.; Humphrey, C. J.; Ducati, C.; Davis, N. J. Emission Properties and Ultrafast Carrier Dynamics of $\mathrm{CsPbCl3}$ Perovskite Nanocrystals. The Journal of Physical Chemistry C 2019, 123, 2651-2657.

(8) Yu, W. W.; Qu, L.; Guo, W.; Peng, X. Experimental determination of the extinction coefficient of CdTe, CdSe, and CdS nanocrystals. Chemistry of Materials 2003, 15, 2854-2860.

(9) Boulesbaa, A.; Issac, A.; Stockwell, D.; Huang, Z.; Huang, J.; Guo, J.; Lian, T. Ultrafast Charge Separation at CdS Quantum Dot/Rhodamine B Molecule Interface. J. Am. 
Chem. Soc. 2007, 129, 15132-15133.

(10)Gollnick, K.; Griesbeck, A. Singlet oxygen photooxygenation of furans: Isolation and reactions of $(4+2)$-cycloaddition products (unsaturated sec.-ozonides). Tetrahedron 1985, 41, 2057-2068.

(11)Yamakoshi, Y.; Umezawa, N.; Ryu, A.; Arakane, K.; Miyata, N.; Goda, Y.; Masumizu, T.; Nagano, T. Active oxygen species generated from photoexcited fullerene (C60) as potential medicines: O2-• versus 1O2. Journal of the American Chemical Society 2003, 125, 12803-12809.

(12)Chen, K.; Yang, W.; Wang, Z.; Iagatti, A.; Bussotti, L.; Foggi, P.; Ji, W.; Zhao, J.; Di Donato, M. Triplet excited state of BODIPY accessed by charge recombination and its application in triplet-triplet annihilation upconversion. The Journal of Physical Chemistry A 2017, 121, 7550-7564. 